\title{
ZUR POSITIONIERUNG DER HUNGAROLOGIE IM 21. JAHRHUNDERT.
}

\author{
ANDREA SEIDLER
}

Universität Wien

andrea.seidler@univie.ac.at

\begin{abstract}
Plenarvortrag anlässlich des VIII. Kongresses der Internationalen Hungarologischen Gesellschaft in Pécs, August 2016
\end{abstract}

Sehr geehrte Festgäste, liebe Kolleginnen und Kollegen,

ich darf Sie alle sehr herzlich bei dieser Eröffnungsfeier zum VIII. Kongress der Internationalen Hungarologischen Gesellschaft begrüßen.

Es ist mir eine besondere Ehre, dass mir die Aufgabe, hier zu sprechen, vom Leitungsgremium der Gesellschaft übertragen wurde. Danke dafür.

Der diesjährige Kongress, es ist der VIII. seiner Art, steht unter dem Motto Kultúra- és tudományköziség - magyarságtudomány a 21. században, es wird also die Frage nach der Positionierung der Hungarologie, der Magyarságtudomány im 21. Jahrhundert gestellt. Die Definition und die Geschichte des Begriffes Hungarologie, den ich hier verwenden möchte, sollte daher unseren Überlegungen vorangestellt werden. Wie Ihnen allen bekannt ist, war der erste Forscher und Lehrer, der diesen Terminus benutzte Robert Gragger, der zu Beginn des 20. Jahrhunderts in Berlin tätig war und 1926, im sehr jungen Alter von nur 39 Jahren verstorben ist. Er hatte unter Hungarologie all jene Wissenschaftsdisziplinen verstanden, die sich mit der Kultur der Ungarn beschäftigte: Literaturwissenschaft, Sprachwissenschaft, Volkskunde, Kunstgeschichte, aber auch Rechtsgeschichte und Wirtschaftsgeschichte. Es handelte sich also um eine sehr modern konzipierte wissenschaftliche Disziplin, die sich fächerübergreifend und raumübergreifend mit Ungarn beschäftigte, wobei der Blick auf den Forschungsgegenstand ein Blick von außen, aus dem Ausland, war. Hungarologen beschäftigen sich dem Graggerschen Verständnis folgend also nicht von Ungarn aus mit dem Fachbereich, sondern vom Ausland aus: Die Dichotomie von Inlands- und Auslandsdisziplin rückte in den Mittelpunkt. Gragger ging von zwei Disziplinen aus, die einander gegenüberstehen und einander doch ergänzen. Wichtig war dabei schon der seiner Zeit vorausgehende Gedanke der Interdisziplinarität, der Überregiona- 
lität und des internationalen Ansatzes. Neben der Fächervielfalt, die die Disziplin ausmachte, spielte bei der Konstituierung des Fachs der Spracherwerb von Beginn an eine zentrale Rolle: Der Hungarologie musste es und muß es auch heute noch unter anderem darum gehen, Interaktions- Verstehens- und Lernprozesse in der Auseinandersetzung mit ungarischsprachigen Diskursen zu ermöglichen. Das Fach „Ungarisch als Fremdsprache“ gehört zu jedem hungarologischen Studium, es muss unterrichtet, gleichzeitig aber aufgewertet und auch weiterentwickelt werden.

Dieser breite Begriff der Hungarologie, der sich mehr oder weniger bis in die 70-er Jahre gehalten hatte, wurde später vor allem von Ungarn - von der ungarischen Politik ausgehend - eingeschränkt und bezog sich nunmehr auf die philologische Disziplin (die Finno-Ugristik als ein großes Teilgebiet dessen) und auf ethnographische Studien.

Ich habe im Zuge der Vorbereitung dieser Rede zahllose Definitionen dieses Begriffs, viele politische Programme, die sich damit beschäftigen und einige von meiner Kollegenschaft verfasste Artikel zu dem Thema gelesen und möchte betonen, dass vor allem um die Wende vom 20. zum 21. Jahrhundert recht viel über das Fach nachgedacht wurde. Holger Fischer hat beispielsweise im Jahr 2002 eine umfassende Darstellung dessen geliefert, was Hungarologie vor allem in Deutschland - in Hamburg - bedeutet, und hat vor allem auch eindrucksvoll gezeigt, was sie in der Praxis zu leisten vermag, aber auch, mit welchen Problemen sie zu kämpfen hat. Der letzte programmatische Beitrag, der auch in der Bibliographie der Gesellschaft aufgelistet ist, stammt aus dem Jahr 2007, er wurde von Kovács Gábor verfasst. Ich selbst komme durch die Verfolgung des Diskurses, aber vor allem durch die persönliche Beobachtung der wissenschaftlichen und universitären Praxis zu einer für mich schlüssigen Ansicht: Es handelt sich dabei um ein Bündel an Wissenschaftsdisziplinen, die sich mit der Geschichte und Kultur Ungarns beschäftigen, wobei stets eine der Disziplinen im Mittelpunkt stehen kann (z. B. die Literaturgeschichte), der Blick aber auf das Gesamte gerichtet bleibt: Sprache, Geschichte, Anthropologie, Kunstgeschichte und modernere Wissenschaftszweige wie Kommunikations- und Medienwissenschaft, sowie Politikwissenschaft. Insofern rückt der Begriff sehr nahe an das Gebiet der sich seit der zweiten Hälfte des 20. Jahrhunderts immer mehr in den Vordergrund spielenden Kulturwissenschaften, die programmatisch ebenfalls wegrücken von der Einzeldisziplin hin zu einer interdisziplinären, überregionalen, gesamtkulturellen Sicht auf die Geschichte der Kultur der Menschheit, der Kultur eines Raumes, der Kultur einer Epoche. Kulturwissenschaften erforschen bekanntlich die materielle und symbolische Dimension von Kulturen und vereinigen die kulturellen Aspekte von Anthropologie, Kunstwissenschaft, Literaturwissenschaft, Musikwissenschaft, Theaterwissenschaft, Medienwissenschaft, Sprachwissenschaft etc. Sie stellen somit einen stark interdisziplinären, sich an der Grenze der 
Human- und Sozialwissenschaften bewegenden Fachbereich dar. Die Kulturwissenschaften arbeiten nach dem holistischen Prinzip: Sie sind auf die Ergebnisse der Einzelwissenschaften angewiesen, versuchen aber durch Reflexion und Kritik die Zusammenhänge in den Blick zu bekommen (Hartmuth Böhme, Reinhard Koselleck: z. B. in Kritik und Krise, 1973)

Das kulturwissenschaftliche Paradigma der Humanwissenschaften kann also geeignet sein, auch der hungarologischen Forschung und Lehre sichere Perspektiven zu eröffnen und sie an breitere, die Tradition der cultural studies produktiv aufgreifende, aber auch weit darüber hinausgehende kulturwissenschaftliche Diskurse anschlussfähig zu machen.

Nun zur Frage: Wie positionieren wir die Hungarologie zu Beginn des 21. Jahrhunderts?

Ich habe in der deutschen Wochenzeitschrift „Die Zeit“ von letzter Woche, genau: in ihrem Supplement „Magazin“, einen kritischen Beitrag zur Entwicklung der Wissenschaften weltweit - aber mit besonderem Fokus auf Europa - gelesen. Aus der Recherche des Journalisten ging hervor, dass es allein in Deutschland ein Studienangebot von 18.000 Fächern gibt. „18.000! Wer soll das alles studieren?", stellte der Redakteur sich und uns die Frage! Die Humanwissenschaften, die Philologien im besonderen, machen dabei nur einen geringen Teil im schier unendlich erscheinenden Feld der Natur- und Sozialwissenschaften aus, deren Ausdifferenzierung in der modernen Wissenschaftslandschaft keine Grenzen zu haben scheint.

Die Frage, die dieser Kongress stellt, ist also mehr als berechtigt! Wenn wir uns diesen Forschungs- und Ausbildungsdschungel vor Augen halten: Wo stehen wir Hungarologen überhaupt zu Beginn des 21. Jahrhunderts? Können wir mithalten mit dem Überangebot an zeitgemäß scheinenden, attraktiven Inhalten anderer Disziplinen. Was können wir, die wir unseren Fächern entsprechend den Blick zum Großteil nach hinten richten - auf historische Zusammenhänge, auf Geschriebenes, Gelebtes/Erlebtes -, was können wir also einer Gesellschaft bieten, die im Hier und Jetzt zu leben scheint und die aus der Geschichte zu lernen kaum noch als wichtig betrachtet? Was einer Gesellschaft, die sich mit historischen Gedächtnis kaum noch auseinandersetzt, der Gedächtnisorte nichts mehr zu sagen scheinen. „Richtig ist, was nachgefragt wird“, resümiert einer der Befragten Experten im Zeit-Artikel über das gegenwärtige Fächerangebot. Aber ist denn die Hungarologie in der heutigen Form noch gefragt?

Lassen Sie uns einige Fakten aufwerfen und Überlegungen zu der Frage des Gefragt-seins anstellen:

Wir alle, die wir hier als VertreterInnen derjenigen humanwissenschaftlichen Disziplinen sitzen, die das breite Forschungsgebiet der Hungarologie ${ }^{1}$ ausmachen, tun dies mit gemischten Gefühlen, denn sonst hätten wir nicht ein Motto gewählt, das förmlich nach Klärung und Neupositionierung schreit: 
Wir alle - von Wien bis Seoul, von Neu Delhi bis Budapest haben mit sehr ähnlichen Herausforderungen zu kämpfen:

- mit abnehmender gesellschaftlicher Anerkennung unserer Fächer,

- mit dem ständigen Legitimationszwang, in dem wir uns befinden,

- mit mangelndem studentischen Interesse an unseren Fächern, das nur teilweise inhaltlich bedingt ist,

- mit der damit einhergehenden niedrigen Dotierung unserer Institutionen in einer Welt, in der die Naturwissenschaften (Technischen Fächer) uns längst den Rang abgelaufen haben,

- mit dem Prestigeschwund der Gesellschaften, Vereine, zivilen Organisationen, die uns - neben den engen institutionellen Bindungen auch die Möglichkeit des Dialogs auf einer breiten, internationalen Ebene geben. Begegnungen wie die heutige, der TeilnehmerInnen aus Dutzenden Ländern angereist sind, zähle ich dazu.

Haben wir im Kampf der Disziplinen um den Fortbestand der Hungarologie schon verloren? Und was können wir überhaupt noch tun, um uns in Zukunft nicht nur über Wasser zu halten, sondern unserer Disziplin, der Hungarologie auch neuen Schwung zu geben? Wie können wir uns zunächst institutionell besser behaupten? Wie können wir unsere Inhalte an die Anforderungen eines modernen Wissenschaftsverständnisses anpassen?

Zunächst zu einer möglichen institutionellen Besserstellung:

Die Hungarologie als BA-MA und PhD-studierbares Fach existiert an einem knappen Dutzend Universitäten weltweit. Ich kann hier weder über die Institute oder Fachbereiche in den USA noch in Asien sprechen. Die europäischen Institute hingegen kenne ich aus eigener Erfahrung sehr gut, ich war an nahezu allen als Erasmus-Lehrerin tätig, an vielen sogar mehrfach. Aber am besten kenne ich natürlich meinen eigenen Fachbereich an der Universität Wien. Es ist bei uns in Wien derzeit möglich, das Fach Hungarologie auf allen drei Stufen des so genannten Bologna-Modells zu studieren: BA, MA, Doktorat. Es umfasst Studien aus dem Bereich der Literaturwissenschaft, der Sprachwissenschaft, der Kulturwissenschaft, der traditionellen Landeskunde sowie den Spracherwerb Ungarisch (und anderer finnisch-ugrischer Sprachen). Die Studienrichtungen werden im institutionellen Rahmen der Abteilung für Finno-Ugristik unterrichtet - dem Wunsch der Hungarologen nach einer Abteilung für Hungarologie an der Universität wurde bislang nicht entsprochen, vielleicht auch, weil er bisher nicht mit Nachdruck geäußert wurde.

Einige Zahlen zum besseren Verständnis: An unserer Abteilung studieren an die 250 Studentinnen - manche von ihnen allerdings Finno-Ugristik oder Fennistik -, die Zahl der AbsolventInnen beträgt jährlich zwischen 15 und 20. Insgesamt doppelt so viel kommen aus allen Studienrichtungen, die allerdings nicht 
zur Hungarologie gezählt werden. Das Interesse an unseren Studienrichtungen schwankt: Wir konnten nach der Wende, zu Beginn der 90-er Jahre des 20. Jahrhunderts einen ungeheuren Zuwachs an Studierenden verzeichnen, die - damals, als es politisch keine Hindernisse mehr gab, die sich ihnen in den Weg stellen würden - aus Ungarn gerne zu uns kamen. Aber auch unter den österreichischen Studierenden erlebte das Fach einen Hype: Der eiserne Vorhang war gefallen, die Möglichkeiten, sich mit dem Nachbarland, das uns künstlich fern gehalten worden war, schienen verlockend. Wer sich kein ganzes Studium der Hungarologie antun wollte, lernte die Sprache - zum Teil auch ganz privater Motivation folgend -, meist aber, weil man sich durch die Kenntnis einer exotischen Sprache, als die das Ungarische galt und gilt, sein Hauptfach aufwertete. Die Universität Wien erweiterte ihr Studienangebot um das Lehramtsfach Ungarisch - ab sofort wurden in Wien Lehrer für das bilinguale Gymnasium im Burgenland ausgebildet. Das Lehramtsstudium ist auch heute noch ein wichtiger Garant für das Fortbestehen des Ungarischen an der Universität Wien.

Die einstige Attraktivität konnte das Fach leider nicht halten: die Verschulung des Universitätsstudiums nach Bologna, der Druck, der auf den Studenten lastet und keinerlei Experimente mit kleinen Fächern zulässt, wenn man an zukünftige Vermittelbarkeit auf dem Arbeitsmarkt denkt, hat die Zahlen nach unten korrigiert. Die Wahl des Studiums ist eine sensible, sie richtet sich unter anderem auch nach dem politischen Prestige des Ziellandes, nach dem Stand der diplomatischen Beziehungen, nach der Presse. So haben wir uns in den letzten Jahren bei den ca. 250 Studierenden eingependelt, von denen oben schon die Rede war. $\mathrm{Ob}$ wir die Zahl halten können, ist ungewiß.

Nun zur Ausrichtung der Hungarologie in Wien:

Die Hungarologie, wie sie in Wien unterrichtet wird, entspricht am ehesten dem Modell einer Auslandsphilologie, die ergänzt wird durch moderne wissenschaftliche Disziplinen und Zugänge, wie die Kulturwissenschaften und die modernen Medienwissenschaften. Oft bestimmt auch der Gastprofessor das Profil unserer speziellen Hungarologie: Im letzten Jahr wurden auch soziologische Fragen der Populärkultur und der modernen Musikgeschichte eingebracht. Im Zentrum unserer Forschung und Lehre steht in Wien auf jeden Fall die Literaturwissenschaft in der weitest möglichen Definition des Begriffes.

Ich sehe die Abgrenzung der Hungarologie von der Finno-Ugristik in Wien als problematisch! Natürlich gehört sie nach meiner Definition des HungarologieBegriffes zur Disziplin in ihrer vielfältigen Ausrichtung dazu! Dennoch: Sieht man sich die Lehrpläne an, so gibt es die Zusammengehörigkeit von Literaturwissenschaft, Kulturwissenschaft und ungarischer Sprachwissenschaft. Die FinnoUgristik führt in Wien traditionell ein eigenes Dasein.

Wo liegen nun die Stärken und wo die Schwächen des Wiener Modells der Hungarologie? 
Beginnen wir mit einem Vergleich, mit einer Schwesterwissenschaft, der Germanistik: Alois Wierlacher, ein Germanist, war in den 80-er Jahren einer der ersten Wissenschaftler, der die Diskussion begonnen hat, dass die Philologien im Ausland unterschiedlich orientiert sein müssten als in den Ländern der Muttersprache. Sie sollten sich als vergleichende Fremdkultur konstituieren. Er bezog sich wie gesagt auf die Germanistik. Wenngleich sein Ansatz zu Beginn sehr umstritten war, führte er doch zu einer umfassenden Neuorientierung des Faches und gleichzeitig zu der Etablierung des Faches Deutsch als Fremdsprache. Und die begonnene Diskussion führte dazu, dass die Fachwelt ein stärkeres Bewusstsein für die Unterschiede zwischen der Germanistik in den deutschsprachigen Ländern und der Germanistik im Ausland entwickelte. Es entstand der Begriff der Auslandsgermanistik, der auch in universitären Einrichtungen gebraucht wurde. Freilich hatten die Vertreter der Disziplin nicht immer Freude an dieser Teilung: Er sollte sich - so der Wunsch - nur auf die nicht-deutschsprachige Unterrichtssituation an den Universitäten beziehen. Die Forscher selbst wollten sich nicht abstempeln lassen: Es gäbe nur eine Germanistik, hieß es. Die Angst um das eigene Image dominierte den Diskurs. Forschung sei ortsunabhängig, lautete die Kritik an dem Konzept.

Worin sah man den Unterschied? Die Auslandsphilologie verfügte über größere wissenschaftliche Neutralität, Distanz und Objektivität, die Inlandsphilologie hingegen behandelte einzelne Themen mit nationalem Identitätsbedürfnis parteilich. Es wurde kritisiert, dass es auf diese Weise einen nationalen Alleinvertretungsanspruch der Inlandphilologie als allein gültige Sprach- und Literaturwissenschaft geben könne. All das mag richtig sein, jedes Argument kann zumindest nachvollzogen werden.

Dennoch: Der analytische Vorsprung informierter Fremdbeschreibung kann nicht geleugnet werden. Es gibt keine zentrale Deutungshoheit, die von der Binnenwissenschaft ausgeht.

Diese Erfahrung ist auch uns Hungarologen nicht fremd: Auch wir verstehen uns als eine Auslandswissenschaft - das Fach Hungarologie gibt es überhaupt nur außerhalb Ungarns -, wir sind Forscherinnen, die sich außerhalb Ungarns mit Themen beschäftigen, die die ungarische Kultur, die ungarische Geschichte, die ungarische Gesellschaft betreffen. Und auch wir Hungarologen, die an ausländischen Universitäten tätig sind, müssen uns zuweilen den Vorwurf gefallen lassen, von weit weg nicht wirklich tief in das Geschehen in Ungarn einblicken zu können - zuweilen auch bedingt durch sprachliche Barrieren. Gibt es also eine Zweiklassengesellschaft, wenn es um die wissenschaftliche Beschäftigung mit Ungarn geht? Und was unsere AbsolventInnen betrifft: Gibt auch da eine Zweiklassengesellschaft was die Studentinnen und AbsolventInnen hungarologischer Fächer angeht? Die Erfahrungen, die ich im Laufe meiner Tätigkeit machte, sagt mir: Ja, die gibt es. Und zwar aus dem einen Grund, dass man weder unse- 
re wissenschaftliche und universitäre Tätigkeit, noch die unserer Studentinnen vergleichen kann mit dem, was Forscherinnen in Ungarn an ihren Universitäten lehren und Studenten lernen. Der Forschungsgegenstand mag derselbe sein, die Perspektive ist aber eine ganz andere.

Die Germanistik hat sich in der Weiterentwicklung des Konzeptes der Auslandphilologie mit dem Terminus der „Interkulturellen Germanistik“ beholfen: Er umfasst die Einsicht, dass es die Disziplin in vielen Ländern der Welt gibt und man von überall mit anderen Augen auf die deutschsprachigen Kernländer blickt. Da die kulturellen Blickwinkel verschieden sind, sieht man auch Verschiedenes (Wieland). Diese globale Vielfalt kultureller Blickwinkel in Perspektivik und Gegenstandkonstitution wissenschaftlicher Arbeit zu erkennen, sowohl in Forschung als auch in Lehre ist das Leitziel interkultureller Philologie. Man könnte hier den Terminus des „Gemeinschafthandelns“ (Max Weber) als ein zentrales Anliegen der Akteure heranziehen.

Die Hungarologie - wenn wir sie in die Richtung interkulturellen Handelns rücken - kann also ebenfalls ein Dachbegriff sein. Die Hungarologie bereichert die Forschung, die im Binnenland auf dem Gebiet der Philologie, der Kultur- und Bildungsgeschichte betrieben wird. Sie kennzeichnet ein Mehr, eine Variante ungarnbezogener Forschung, die durch die regionale Fremdkultur, von der aus sie betrieben wird, bereichert wird. Die Hungarologie nimmt die Kulturengebundenheit sowohl in Forschung wie in Lehre aber auch - und das scheint mir wichtig - in der Wissenschaftsorganisation ernst.

Sie hält die kulturelle Vielfalt der wissenschaftlichen Interessen, Fragestellungen und Annäherungsweisen nicht für einen Nachteil, sondern für einen Vorteil der Erkenntnisarbeit. Sie konstituiert sich in Forschung und Lehre als Teil interkultureller Kommunikation mit dem Ziel, im Dialog der Kulturen praktisch werden und zu Problemen vorzustoßen, die wir heute alle gemeinsam haben.

Die Herausforderung des Faches ist es also, diesen Blick von außen zu verstärken und - das scheint mir ebenfalls wichtig - auch die Fächergrenzen aufzuweichen. Wie bereits erwähnt, ist die Hungarologie in Wien eng an das Studium der Philologie gebunden, es könnte aber auch ein anderes Konzept im Mittelpunkt stehen. Die Kulturwissenschaften, die Medienwissenschaften, aber auch soziologische Disziplinen könnten ebenso zentrale Funktionen übernehmen, die strengen Grenzen der Einzeldisziplinen aufgeweicht werden. Wäre diese Einbettung gegeben, so könnte auch neues Interesse an der Hungarologie entstehen, und zwar nicht nur im Bereich der Forschung, sondern auch im Unterrichtsbereich. Der Schwund an der Zahl der Studierenden, mit dem die ausländischen hungarologischen Institute, Seminare, Abteilungen zu kämpfen haben zeigt uns am drastischsten, dass hier ein Neu- und Umdenken gefordert ist. Und dieses Neudenken kann sich nur an der Analyse dessen orientieren, was die Gesellschaft von uns erwartet. Wichtig ist die Einbettung unseres Faches in ein zentral- oder 
gesamteuropäisches - ja auch globales - Weltverständnis. Wir müssen uns dessen gewärtig sein, dass Bildung heute etwas anderes bedeutet und fordert als noch vor 20 Jahren. Die Zugänge zur Forschung haben sich grundsätzlich geändert, Theorien haben auf eine vollkommen neue Ausrichtung der Welt reagiert, die nicht mehr in Grenzen sondern eventuell noch in Regionen denken will.

Setzen wir also unsere eigenen Regionen in Kommunikation mit der Kultur, die uns alle, die wir hier versammelt sind, als Forscherinnen interessiert: In einen Diskurs mit Ungarn - mit dem Ungarn der Gegenwart aber auch dem Ungarn der Vergangenheit, dessen Grenzen anders verliefen, als die heutigen. Lassen Sie uns dieses Mehr, dieses Andere, zielorientiert einbringen in eine Wissenschaft, die über Grenzen hinweg, interkulturell agiert. Und lassen Sie uns stets daran denken, dass wir eine von 18.000 Studienrichtungen sind: studiert wird, wie oben zitiert, was gefragt ist. Bieten wir den Studierenden eine modern ausgerichtete, kritische Hungarologie, die sich auch in der beruflichen Praxis bewährt und verfolgen wir mit unseren KollegInnen im Binnenland Ungarn einen zeitgemäßen, den neuen Wissenschaften gerecht werdenden Diskurs, um Fragen der Vergangenheit, der Gegenwart aber auch der Zukunft gemeinsam zu beantworten.

In diesem Sinne wünsche ich uns allen anregende Kongresstage. Das Programm ist breit gefächert, es ist für jede und jeden etwas dabei! Und lassen sie uns außerhalb der Vorträge auch zusammenkommen, um eben diese Fragen einer zukünftigen Gestaltung der Hungarologie zu erörtern. Jede Erfahrung, jede Meinung ist wertvoll.

Anmerkung

1 http://www.hitelfolyoirat.hu/sites/default/files/pdf/104-108.pdf 